\section{New Theory}

ISSN: 2149-1402
35 (2021) 72-90

Journal of New Theory

https://dergipark.org.tr/en/pub/jnt

Open Access

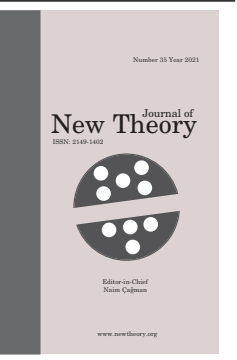

\title{
Bimultiplications and Annihilators of Crossed Modules in Associative Algebras
}

\author{
Ummahan Ege Arslan ${ }^{1}$ (D), Serdar Hürmetli ${ }^{2}$
}

\begin{abstract}
Article History
Received: 29 Mar 2021

Abstract - In this paper, we present a generalization of the concept of the bimultiplication algebra by defining the bimultiplication of crossed modules in associative algebras. Using this structure, we construct the actor of a crossed module in associaAccepted: 19 Jun 2021

Published: 30 Jun 2021

Research Article tive algebras, and we obtain annihilators of crossed modules as the kernel of a crossed module morphism from a crossed module to its actor. Moreover, we construct a link between this morphism and a crossed square, two-dimensional crossed module.
\end{abstract}

Keywords - Bimultiplication crossed module, actor crossed module, annihilator of crossed module, crossed square

Mathematics Subject Classification (2020) - 16Y99, 18A05

\section{Introduction}

In the case of groups, the description of an action is provided by the automorphism group. The action of any group $\mathrm{A}$ on itself is given by a group homomorphism $A \longrightarrow A u t(A)$. In some other algebraic contexts, automorphism structure is not enough to give an action. The set of automorphisms of an associative algebra is not usually an algebra. For associative algebra case, the bimultiplication algebra $\operatorname{Bim}(G)$ of an associative algebra $G$ which is defined by Lane [1] will accomplish the role of an automorphism group.

The concept of a crossed module for groups is defined in $[2,3]$ by Whitehead. The commutative algebra version of crossed modules is mentioned in a different name, by Lichtenbaum and Schlessinger [4] also, the paper of Gerstenhaber [5] contains the notion of crossed modules in commutative algebras. The notion of crossed modules in associative algebras is defined by Dedecker and Lue in [6].

In [7], Norrie improved Lue's work [8], and she defined the concept of an actor of crossed modules for groups as analogue of an automorphism group. For commutative algebras, Arvasi and the first author explained that this notion is related to the multiplication algebra in [9]. In this paper based on the second author's $\mathrm{PhD}$ dissertation [10], our starting point is the generalization of the bimultiplication algebra. For this, we define the bimultiplication of crossed modules for associative algebras and give details of the construction of actor crossed module for associative algebras via this context. The notion of actor crossed module is also studied simultaneously by Boyacı et al. in [11] as the split extension classifier of a crossed module. By constructing a morphism under certain conditions from a crossed module and its actor, we get the action of any crossed modules in associative algebras on itself. Since the annihilator of an algebra $A$ is given by the kernel of algebra homomorphism $A \longrightarrow \operatorname{Bim}(A)$,

\footnotetext{
${ }^{1}$ uege@ogu.edu.tr (Corresponding Author); ${ }^{2}$ serdar_hurmetli@hotmail.com

${ }^{1,2}$ Department of Mathematics and Computer Science, Faculty of Science and Letters, Eskişehir Osmangazi University, Eskişehir, Turkey
} 
we have a similar notion for a crossed modules in associative algebras via the kernel of the abovementioned morphism between a crossed module and its actor. Also, while we get $A \longrightarrow \operatorname{Bim}(A)$ crossed module as a two-dimensional associative algebra via an algebra $A$, we can have a crossed square as a two-dimensional crossed module via an appropriate crossed module morphism.

\section{Preliminary}

Conventions: Throughout this paper, it is supposed that $K$ is a fixed commutative ring with identity $1 \neq 0, K$-algebra $G$ is a unitary $K$-bimodule $G$ equipped with a $K$-bilinear associative multiplication and also if $G$ is a $K$-algebra, then a $G$-algebra $C$ is a $G$-bimodule equipped with a $G$-bilinear associative multiplication. $C$ will not necessarily have identities.

We recall the definition of a crossed module in associative algebras [12].

Definition 2.1. A crossed module in associative $K$-algebras is a morphism from a $G$-algebra $C$ to a $K$-algebra $G, \delta: C \longrightarrow G$, with two-sided actions of $G$ on $C$ satisfying

CM1) $\delta(g \cdot c)=g \delta(c)$ and $\delta(c \cdot g)=\delta(c) g$

$\mathrm{CM} 2) \delta c \cdot c^{\prime}=c c^{\prime}$ and $c \cdot \delta c^{\prime}=c c^{\prime}$

for all $c \in C, g \in G$. It is denoted by $(C, G, \delta)$.

The following are some standard examples of crossed modules in associative algebras:

i. An inclusion map $I \hookrightarrow G$ is a crossed module, where $I$ is any two-sided ideal in $G$. On the other hand, let $\delta: C \longrightarrow G$ be any crossed module. Then, we get that $\delta(C)$ is a two sided ideal in $G$ by CM1.

ii. Any $G$-bimodule $M$ has a $G$-algebra structure with zero multiplication. Thus, we get the crossed module $\mathbf{0}: M \rightarrow G, \mathbf{0}(m)=0$. Conversely, the kernel of crossed module $\delta: N \longrightarrow G$ is an $G / \delta(N)$-bimodule.

Thus, the concept of crossed modules in associative algebras is a generalisation the concepts both of a two-sided ideal and that of a bimodule over an algebra. Also, any associative algebra is considered as a crossed modules by identity map $I d: G \longrightarrow G$.

Definition 2.2. A pair $(f, \phi)$ of $G$-algebra morphisms $f: C \longrightarrow C^{\prime}, \phi: G \longrightarrow G^{\prime}$ such that

$$
f(g \cdot c)=\phi(g) \cdot f(c) \quad \text { and } \quad f(c \cdot g)=f(c) \cdot \phi(g)
$$

for $c \in C, g \in G$ is called a morphism of crossed modules from $(C, G, \delta)$ to $\left(C^{\prime}, G^{\prime}, \delta^{\prime}\right)$.

Thus, we get the category AssXMod of crossed modules in associative algebras.

The kernel of $(f, \phi)$ and the image $\operatorname{Im}(f, \phi)$ are defined by $(\operatorname{ker} f, \operatorname{ker} \phi, \delta)$ and $\left(\operatorname{Imf}, \operatorname{Im} \phi, \delta^{\prime}\right)$, respectively.

Definition 2.3. A crossed module $\left(C^{\prime}, G^{\prime}, \delta^{\prime}\right)$ is called a subcrossed module of a crossed module $(C, G, \delta)$ if the following conditions are satisfied:

i. $C^{\prime}$ and $G^{\prime}$ are the subalgebra of $C$ and $G$, respectively

ii. $\delta^{\prime}=\left.\delta\right|_{C^{\prime}}$

iii. The action of $G^{\prime}$ on $C^{\prime}$ inherits from the action of $G$ on $C$

The image $\operatorname{Im}(f, \phi)$ of $(f, \phi)$ is the subcrossed module $\left(\operatorname{Imf}, \operatorname{Im} \phi, \delta^{\prime}\right)$ of $(C, G, \delta)$. 
Definition 2.4. A subcrossed module $\left(C^{\prime}, G^{\prime}, \delta^{\prime}\right)$ of $(C, G, \delta)$ is called an ideal if

i. $G^{\prime}$ is an two sided ideal of $G$

ii. $g \cdot c^{\prime} \in C^{\prime}$

iii. $g^{\prime} \cdot c \in C^{\prime}$

for all $g \in G, g^{\prime} \in G^{\prime} c \in C, c^{\prime} \in C^{\prime}$.

If $(f, \phi):(C, G, \delta) \longrightarrow\left(C^{\prime}, G^{\prime}, \delta^{\prime}\right)$ is any crossed module morphism, (ker $f$, ker $\left.\phi, \delta\right)$ is an ideal of $(C, R, \delta)$ and $\left(\operatorname{Imf}, \operatorname{Im} \phi, \delta^{\prime}\right)$ is a subcrossed module of $\left(C^{\prime}, G^{\prime}, \delta^{\prime}\right)$.

Definition 2.5. Let $\left(R, S, \delta^{\prime}\right)$ be an ideal of $(C, G, \delta)$. Then, $\bar{\delta}: C / R \longrightarrow G / S, \bar{\delta}(c+R)=\delta(c)+S$ is a crossed module with $(g+S) \cdot(c+R)=(g \cdot c)+R$ and $(c+R) \cdot(g+S)=(c \cdot g)+R$. It is called the quotient crossed module of $(C, G, \delta)$ by $\left(R, S, \delta^{\prime}\right)$ and denoted by $\frac{(C, G, \delta)}{\left(R, S, \delta^{\prime}\right)}$.

\section{Actor Crossed Modules in Associative Algebras}

\subsection{A Bimultiplier of a Crossed Module}

We recall the structure of the $R$-algebra of bimultipliers of $C, \operatorname{Bim}(C)[1,13] . \operatorname{Bim}(C)$ consists of all pairs $(\gamma, \delta)$ of $R$-linear mappings $\gamma, \delta: C \rightarrow C$ such that

$$
\begin{gathered}
\gamma\left(c c^{\prime}\right)=\gamma(c) \cdot c^{\prime}, \\
\delta\left(c c^{\prime}\right)=c \cdot \delta\left(c^{\prime}\right),
\end{gathered}
$$

and

$$
c \cdot \gamma\left(c^{\prime}\right)=\delta(c) \cdot c^{\prime}
$$

It has an $R$-module structure and a product

$$
(\gamma, \delta) \cdot\left(\gamma^{\prime}, \delta^{\prime}\right)=\left(\gamma \circ \gamma^{\prime}, \delta^{\prime} \circ \delta\right)
$$

Suppose that $\operatorname{Ann}(C)=0$ or $C^{2}=C$. Then, $\operatorname{Bim}(C)$ acts on $C$ by

$$
\begin{array}{llll}
\operatorname{Bim}(C) \times C & \rightarrow C ; & ((\gamma, \delta), c) & \mapsto \gamma(c), \\
C \times \operatorname{Bim}(C) & \rightarrow C ; & (c,(\gamma, \delta)) & \mapsto \delta(c)
\end{array}
$$

and there is a

$$
\begin{aligned}
\mu: C & \longrightarrow \operatorname{Bim}(C) \\
c & \longmapsto\left(\gamma_{c}, \delta_{c}\right)
\end{aligned}
$$

with

$$
\gamma_{c}(x)=c \cdot x \quad \text { and } \quad \delta_{c}(x)=x \cdot c
$$

We give the following result from their work of Lavendhomme and Lucas [14].

Proposition 3.1. Let $G$ be a $K$-algebra such that Ann $(G)=0$ or $G^{2}=G$. Then, $(G, \operatorname{Bim}(G),(\gamma, \sigma))$ is a crossed module.

Proof. $\operatorname{Bim}(G)$ acts on $G$ by

$$
\begin{aligned}
\operatorname{Bim}(G) \times G & \longrightarrow G \\
\left(\left(\gamma^{\prime}, \sigma^{\prime}\right), g\right) & \longmapsto \gamma^{\prime}(g)
\end{aligned} \quad \text { and } \begin{aligned}
G \times \operatorname{Bim}(G) & \longrightarrow G \\
\left(g,\left(\gamma^{\prime}, \sigma^{\prime}\right)\right) & \longmapsto \sigma^{\prime}(g)
\end{aligned}
$$

and there is a $(\gamma, \sigma): G \longrightarrow \operatorname{Bim}(G)$ defined by $(\gamma, \sigma)(g)=\left(\gamma_{g}^{\prime}, \sigma_{g}^{\prime}\right)$ with $\gamma_{g}^{\prime}\left(g^{\prime}\right)=g g^{\prime}$ and $\sigma_{g}^{\prime}\left(g^{\prime}\right)=g^{\prime} g$ for all $g, g^{\prime} \in G$. 
CM1)

$$
\begin{aligned}
(\gamma, \sigma)\left(\left(\gamma^{\prime}, \sigma^{\prime}\right) \cdot g\right) & =(\gamma, \sigma)\left(\gamma^{\prime}(g)\right) \\
& =\left(\gamma_{\gamma^{\prime}(g)}^{\prime}, \sigma_{\gamma^{\prime}(g)}^{\prime}\right) \\
& \stackrel{(*)}{=}\left(\gamma^{\prime} \gamma^{\prime}(g), \sigma^{\prime}(g) \sigma^{\prime}\right) \\
= & \left(\gamma^{\prime}, \sigma^{\prime}\right)\left(\gamma^{\prime}(g), \sigma^{\prime}(g)\right) \\
= & \left(\gamma^{\prime}, \sigma^{\prime}\right)((\gamma, \sigma)(g))
\end{aligned}
$$

$(*)$

$$
\begin{array}{rlrl}
\left(\gamma_{\gamma^{\prime}(g)}^{\prime}\right)\left(g^{\prime}\right) & =\gamma^{\prime}(g) g^{\prime} & \left(\sigma_{\gamma^{\prime}(g)}^{\prime}\right)\left(g^{\prime}\right) & =g^{\prime} \gamma^{\prime}(g) \\
& =\gamma^{\prime}\left(g g^{\prime}\right) & & =\sigma^{\prime}\left(g^{\prime}\right) g \\
& =\gamma^{\prime}\left(\gamma_{g}^{\prime}\left(g^{\prime}\right)\right) & & =\sigma_{g}^{\prime}\left(\sigma^{\prime}\left(g^{\prime}\right)\right) \\
& =\left(\gamma^{\prime} \gamma^{\prime}(g)\right)\left(g^{\prime}\right) & & \\
(\gamma, \sigma)\left(g \cdot\left(\gamma^{\prime}, \sigma^{\prime}\right)\right) & = & (\gamma, \sigma)\left(\sigma^{\prime}(g)\right) & \\
& =\left(\gamma_{\sigma^{\prime}(g)}^{\prime}, \sigma_{\sigma^{\prime}(g)}^{\prime}\right) & \\
& \stackrel{(*)}{=}\left(\gamma^{\prime}(g) \gamma^{\prime}, \sigma^{\prime} \sigma^{\prime}(g)\right) \\
& =\left(\gamma^{\prime}(g), \sigma^{\prime}(g)\right)\left(\gamma^{\prime}, \sigma^{\prime}\right) & \\
& =((\gamma, \sigma)(g))\left(\gamma^{\prime}, \sigma^{\prime}\right)
\end{array}
$$

$(*) \quad\left(\gamma_{\sigma^{\prime}(g)}^{\prime}\right)\left(g^{\prime}\right)=\sigma^{\prime}(g) g^{\prime}$

$$
\begin{aligned}
\left(\sigma_{\sigma^{\prime}(g)}^{\prime}\right)\left(g^{\prime}\right) & =g^{\prime} \sigma^{\prime}(g) \\
& =\sigma^{\prime}\left(g^{\prime} g\right) \\
& =\sigma^{\prime}\left(\sigma_{g}^{\prime}\left(g^{\prime}\right)\right) \\
& =\left(\sigma^{\prime} \sigma^{\prime}(g)\right)\left(g^{\prime}\right)
\end{aligned}
$$$$
=g \gamma^{\prime}\left(g^{\prime}\right)
$$$$
=\gamma_{g}^{\prime}\left(\gamma^{\prime}\left(g^{\prime}\right)\right)
$$$$
=\left(\gamma^{\prime}(g) \gamma^{\prime}\right)\left(g^{\prime}\right)
$$

CM2)

$$
\begin{aligned}
((\gamma, \sigma)(g)) \cdot g^{\prime} & =\left(\gamma_{g}^{\prime}, \sigma_{g}^{\prime}\right) \cdot g^{\prime} \\
& =\gamma_{g}^{\prime}\left(g^{\prime}\right) \\
& =g g^{\prime}
\end{aligned}
$$$$
g \cdot\left((\gamma, \sigma)\left(g^{\prime}\right)\right)=g \cdot\left(\gamma_{g^{\prime}}^{\prime}, \sigma_{g^{\prime}}^{\prime}\right)
$$$$
=\sigma_{g^{\prime}}^{\prime}(g)
$$$$
=g g^{\prime}
$$

Definition 3.2. A bimultiplier of the crossed module $\mu: T \longrightarrow L$ is a pair of

$$
(\lambda, \chi)=\left((\Theta, \Xi),\left(\Theta^{\prime}, \Xi^{\prime}\right)\right):(T, L, \mu) \longrightarrow(T, L, \mu)
$$

satisfying the following conditions:

i. $(\lambda=(\Theta, \Xi): T \longrightarrow T) \in \operatorname{Bim}(T) \quad$ and $\quad\left(\chi=\left(\Theta^{\prime}, \Xi^{\prime}\right): L \longrightarrow L\right) \in \operatorname{Bim}(L)$

ii.

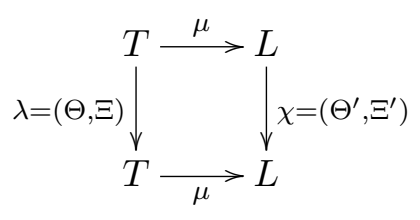

$\chi \mu=\mu \lambda \quad\left(\Theta^{\prime} \mu=\mu \Theta, \Xi^{\prime} \mu=\mu \Xi\right)$

and 
iii. For $l \in L, t \in T$,

$$
\begin{aligned}
& \Theta(l \cdot t)=\Theta^{\prime}(l) \cdot t \\
& \Theta(t \cdot l)=\Theta(t) \cdot l \\
& \Xi(l \cdot t)=l \cdot \Xi(t) \\
& \Xi(t \cdot l)=t \cdot \Xi^{\prime}(l) \\
& l \cdot \Theta(t)=\Xi^{\prime}(l) \cdot t \\
& \Xi(t) \cdot l=t \cdot \Theta^{\prime}(l)
\end{aligned}
$$

The set of bimultipliers of the crossed module $\mu: T \longrightarrow L$ will be denoted by $\operatorname{Bim}(T, L, \mu)$.

Proposition 3.3. The set $\operatorname{Bim}(T, L, \mu)$ has an associative $K$-algebra structure with following operations:

$$
\begin{array}{ll}
+) & \left((\Theta, \Xi),\left(\Theta^{\prime}, \Xi^{\prime}\right)\right)+\left((\bar{\Theta}, \bar{\Xi}),\left(\bar{\Theta}^{\prime}, \bar{\Xi}^{\prime}\right)\right)=\left((\Theta+\bar{\Theta}, \Xi+\bar{\Xi}),\left(\Theta^{\prime}+\bar{\Theta}^{\prime}, \Xi^{\prime}+\bar{\Xi}^{\prime}\right)\right. \\
\text {.) } & k\left((\Theta, \Xi),\left(\Theta^{\prime}, \Xi^{\prime}\right)\right)=(k \Theta, k \Xi),\left(k \Theta^{\prime}, k \Xi^{\prime}\right) \\
\circ) & \left((\Theta, \Xi),\left(\Theta^{\prime}, \Xi^{\prime}\right)\right) \circ\left((\bar{\Theta}, \bar{\Xi}),\left(\bar{\Theta}^{\prime}, \bar{\Xi}^{\prime}\right)\right)=\left((\Theta \circ \bar{\Theta}, \bar{\Xi} \circ \Xi),\left(\Theta^{\prime} \circ \bar{\Theta}^{\prime}, \bar{\Xi}^{\prime} \circ \bar{\Xi}\right)\right)
\end{array}
$$

Proof. See for details [10].

Definition 3.4. Let $(T, L, \mu)$ be a crossed module. $\mathcal{U}(L, T)$ is the set of pair maps $\left(\beta_{1}, \beta_{2}\right)$ such that

$$
\begin{aligned}
\beta_{1}\left(l_{1} l_{2}\right) & =\beta_{1}\left(l_{1}\right) \cdot l_{2} \\
\beta_{2}\left(l_{1} l_{2}\right) & =l_{1} \cdot \beta_{2}\left(l_{2}\right) \\
l_{1} \cdot \beta_{1}\left(l_{2}\right) & =\beta_{2}\left(l_{1}\right) \cdot l_{2}
\end{aligned}
$$

for all $l_{1}, l_{2} \in L$, where $\beta_{1}, \beta_{2}: L \rightarrow T$, are $K$-linear maps.

$\mathcal{U}(L, T)$ has an associative $K$-algebra structure with the following operations:

$$
\begin{array}{ll}
+) & \left(\left(\beta_{1}, \beta_{2}\right)+\left(\theta_{1}, \theta_{2}\right)\right)=\left(\beta_{1}+\theta_{1}, \beta_{2}+\theta_{2}\right) \\
\text {.) } & k\left(\beta_{1}, \beta_{2}\right)=\left(k \beta_{1}, k \beta_{2}\right) \\
\text { ○) } & \left(\left(\beta_{1}, \beta_{2}\right) \circ\left(\theta_{1}, \theta_{2}\right)\right)=\left(\beta_{1} \mu \theta_{1}, \theta_{2} \mu \beta_{2}\right)
\end{array}
$$

Proposition 3.5. Let $(T, L, \mu)$ be a crossed module and $\left(\beta_{1}, \beta_{2}\right) \in \mathcal{U}(L, T)$. Each such $K$-linear maps $\beta_{1}, \beta_{2}: L \longrightarrow T$ defines bimultipliers $\left(\Theta_{\beta}, \Xi_{\beta}\right),\left(\Theta_{\beta}^{\prime}, \Xi_{\beta}^{\prime}\right)$ of $T$ and $L$ respectively, given by $\Theta_{\beta}=\beta_{1} \mu$, $\Xi_{\beta}=\beta_{2} \mu, \Theta_{\beta}^{\prime}=\mu \beta_{1}, \Xi_{\beta}^{\prime}=\mu \beta_{2}$.

Proof.

$$
\begin{aligned}
\Theta_{\beta}\left(t t^{\prime}\right) & =\beta_{1}\left(\mu\left(t t^{\prime}\right)\right) \\
& =\beta_{1}\left(\mu(t) \mu\left(t^{\prime}\right)\right) \\
& =\beta_{1}(\mu(t)) \cdot \mu\left(t^{\prime}\right) \\
& =\Theta_{\beta}(t) \cdot \mu\left(t^{\prime}\right) \\
& =\Theta_{\beta}(t) \cdot t^{\prime} \\
\Xi_{\beta}\left(t t^{\prime}\right) & =\beta_{2}\left(\mu\left(t t^{\prime}\right)\right) \\
& =\beta_{2}\left(\mu(t) \mu\left(t^{\prime}\right)\right) \\
& =\mu(t) \cdot \beta_{2}\left(\mu\left(t^{\prime}\right)\right) \\
& =\mu(t) \cdot \Xi_{\beta}\left(t^{\prime}\right) \\
& =t \cdot \Xi_{\beta}\left(t^{\prime}\right)
\end{aligned}
$$




$$
\begin{aligned}
t \Theta_{\beta}\left(t^{\prime}\right) & =t \beta_{1}\left(\mu\left(t^{\prime}\right)\right) \\
& =\mu(t) \cdot \beta_{1}\left(\mu\left(t^{\prime}\right)\right) \\
& =\beta_{2}(\mu(t)) \cdot \mu\left(t^{\prime}\right) \\
& =\beta_{2}(\mu(t)) t^{\prime} \\
& =\Xi_{\beta}(t) t^{\prime}
\end{aligned}
$$

for $t, t^{\prime} \in T$. Thus, $\left(\Theta_{\beta}, \Xi_{\beta}\right) \in \operatorname{Bim}(T)$. Similarly, $\left(\Theta_{\beta}^{\prime}, \Xi_{\beta}^{\prime}\right) \in \operatorname{Bim}(L)$.

Proposition 3.6. The bimultipliers $\lambda_{\beta}=\left(\Theta_{\beta}, \Xi_{\beta}\right)$ of $\mathrm{T}$ and $\chi_{\beta}=\left(\Theta_{\beta}^{\prime}, \Xi_{\beta}^{\prime}\right)$ of L satisfy the following conditions:

$i$.

$$
\begin{aligned}
& \Theta_{\beta} \beta_{1}=\beta_{1} \mu \beta_{1}=\beta_{1} \Theta_{\beta}^{\prime} \quad \text { and } \quad \Xi_{\beta} \beta_{2}=\beta_{2} \mu \beta_{2}=\beta_{2} \Xi_{\beta}^{\prime} \\
& \Theta_{\beta} \beta_{2}=\beta_{1} \mu \beta_{2}=\beta_{1} \Xi_{\beta}^{\prime} \quad \text { and } \quad \Xi_{\beta} \beta_{1}=\beta_{2} \mu \beta_{1}=\beta_{2} \Theta_{\beta}^{\prime}
\end{aligned}
$$

ii. The following diagram

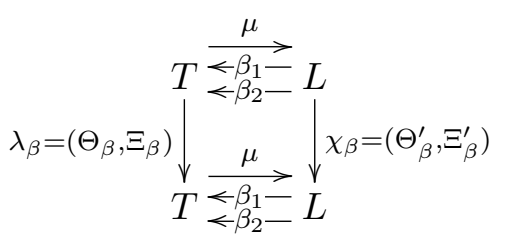

is commutative, that is

$$
\begin{aligned}
& \mu \Theta_{\beta}=\mu\left(\beta_{1} \mu\right)=\left(\mu \beta_{1}\right) \mu=\Theta_{\beta}^{\prime} \mu \\
& \mu \Xi_{\beta}=\mu\left(\beta_{2} \mu\right)=\left(\mu \beta_{2}\right) \mu=\Xi_{\beta}^{\prime} \mu
\end{aligned}
$$

iii.

$$
\left(\lambda_{\beta}, \chi_{\beta}\right)=\left(\left(\Theta_{\beta}, \Xi_{\beta}\right),\left(\Theta_{\beta}^{\prime}, \Xi_{\beta}^{\prime}\right)\right) \in \operatorname{Bim}(T, L, \mu)
$$

Proposition 3.7. If $\mu: T \longrightarrow L$ is a crossed module,then

$$
\begin{aligned}
\Gamma: U(L, T) & \longrightarrow \operatorname{Bim}(T) \\
\left(\beta_{1}, \beta_{2}\right) & \longmapsto\left(\Theta_{\beta}, \Xi_{\beta}\right)=\left(\beta_{1} \mu, \beta_{2} \mu\right) \\
\Phi: U(L, T) & \longrightarrow \operatorname{Bim}(L) \\
\left(\beta_{1}, \beta_{2}\right) & \longmapsto\left(\Theta_{\beta}^{\prime}, \Xi_{\beta}^{\prime}\right)=\left(\mu \beta_{1}, \mu \beta_{2}\right)
\end{aligned}
$$

are algebra morphisms.

Lemma 3.8. Let $\mu: T \longrightarrow L$ be a crossed module. Then, the left and right actions of $\operatorname{Bim}(T, L, \mu)$ on $\mathrm{U}(\mathrm{L}, \mathrm{T})$ is given by

$$
\begin{array}{ccc}
\operatorname{Bim}(T, L, \mu) \times U(L, T) & \longrightarrow U(L, T) \\
\left((\lambda, \chi),\left(\theta_{1}, \theta_{2}\right)\right) & \longmapsto & \left(\Theta \theta_{1}, \theta_{2} \Xi^{\prime}\right) \\
U(L, T) \times \operatorname{Bim}(T, L, \mu) & \longrightarrow & U(L, T) \\
\left(\left(\theta_{1}, \theta_{2}\right),(\lambda, \chi)\right) & \longmapsto & \left(\theta_{1} \Theta^{\prime}, \Xi \theta_{2}\right)
\end{array}
$$

where $(\lambda, \chi)=\left((\Theta, \Xi),\left(\Theta^{\prime}, \Xi^{\prime}\right)\right)$

Proof. Since

$$
\begin{aligned}
\left(\Theta \theta_{1}\right)\left(l l^{\prime}\right) & =\Theta\left(\theta_{1}\left(l l^{\prime}\right)\right) & \left(\theta_{2} \Xi^{\prime}\right)\left(l l^{\prime}\right) & =\theta_{2}\left(\Xi^{\prime}\left(l l^{\prime}\right)\right) \\
& =\Theta\left(\theta_{1}(l) \cdot l^{\prime}\right) & & \theta_{2}\left(l \Xi^{\prime}\left(l^{\prime}\right)\right) \\
& =\Theta\left(\theta_{1}(l)\right) \cdot l^{\prime} & & =l \cdot \theta_{2}\left(\Xi^{\prime}\left(l^{\prime}\right)\right) \\
& \left.=\left(\left(\Theta \theta_{1}\right)(l)\right) \cdot l^{\prime}\right) & & =l \cdot\left(\left(\theta_{2} \Xi^{\prime}\right)\left(l^{\prime}\right)\right)
\end{aligned}
$$




$$
\begin{aligned}
l \cdot\left(\left(\Theta \theta_{1}\right)\left(l^{\prime}\right)\right) & =l \cdot\left(\Theta\left(\theta_{1}\left(l^{\prime}\right)\right)\right) \\
& =\Xi^{\prime}(l) \cdot \theta_{1}\left(l^{\prime}\right) \\
& =\theta_{2}\left(\Xi^{\prime}(l)\right) \cdot l^{\prime} \\
& =\left(\left(\theta_{2} \Xi^{\prime}\right)(l)\right) \cdot l^{\prime}
\end{aligned}
$$

the left action of $\operatorname{Bim}(T, L, \mu)$ on $\mathrm{U}(\mathrm{L}, \mathrm{T})$ is well defined. Since

$$
\begin{aligned}
& \left(\theta_{1} \Theta^{\prime}\right)\left(l l^{\prime}\right)=\theta_{1}\left(\Theta^{\prime}\left(l l^{\prime}\right)\right) \\
& \left(\Xi \theta_{2}\right)\left(l l^{\prime}\right)=\Xi\left(\theta_{2}\left(l l^{\prime}\right)\right) \\
& =\theta_{1}\left(\Theta^{\prime}(l) l^{\prime}\right) \\
& =\Xi\left(l \cdot \theta_{2}\left(l^{\prime}\right)\right) \\
& =\left(\theta_{1}\left(\Theta^{\prime}(l)\right)\right) \cdot l^{\prime} \\
& =l \cdot \Xi\left(\theta_{2}\left(l^{\prime}\right)\right) \\
& =\left(\left(\theta_{1} \Theta^{\prime}\right)(l)\right) \cdot l^{\prime} \\
& =l \cdot\left(\left(\Xi \theta_{2}\right)\left(l^{\prime}\right)\right) \\
& l \cdot\left(\left(\theta_{1} \Theta^{\prime}\right)\left(l^{\prime}\right)\right)=l \cdot\left(\theta_{1}\left(\Theta^{\prime}\left(l^{\prime}\right)\right)\right) \\
& =\theta_{2}(l) \cdot\left(\Theta^{\prime}\left(l^{\prime}\right)\right. \\
& =\Xi\left(\theta_{2}(l)\right) \cdot l^{\prime} \\
& =\left(\left(\Xi \theta_{2}\right)(l)\right) \cdot l^{\prime}
\end{aligned}
$$

the right action of $\operatorname{Bim}(T, L, \mu)$ on $\mathrm{U}(\mathrm{L}, \mathrm{T})$ is well defined.

Moreover, one sees that associative algebra action conditions are satisfied by routine calculations. (See for details $[10]$ )

The next theorem begins the analysis of the associative algebra version of the actor structure.

Theorem 3.9. If $\mu: T \longrightarrow L$ is a crossed module, then the morphism

$$
\triangle: U(L, T) \rightarrow \operatorname{Bim}(T, L, \mu)
$$

is a crossed module given by

$$
\triangle\left(\beta_{1}, \beta_{2}\right)=\left(\left(\Theta_{\beta_{1}}, \Xi_{\beta_{2}}\right),\left(\Theta_{\beta_{1}}^{\prime}, \Xi_{\beta_{2}}^{\prime}\right)\right)=\left(\left(\beta_{1} \mu, \beta_{2} \mu\right),\left(\mu \beta_{1}, \mu \beta_{2}\right)\right) .
$$

Proof. The right and left actions of $\operatorname{Bim}(T, L, \mu)$ on $\mathcal{U}(L, T)$ are given by

$$
\left((\Theta, \Xi),\left(\Theta^{\prime}, \Xi^{\prime}\right)\right) \cdot\left(\theta_{1}, \theta_{2}\right)=\left(\Theta \theta_{1}, \theta_{2} \Xi^{\prime}\right)
$$

and

$$
\left(\theta_{1}, \theta_{2}\right) \cdot\left((\Theta, \Xi),\left(\Theta^{\prime}, \Xi^{\prime}\right)\right)=\left(\theta_{1} \Theta^{\prime}, \Xi \theta_{2}\right)
$$

as mentioned before. We need to check the crossed module axioms:

For $\left((\Theta, \Xi),\left(\Theta^{\prime}, \Xi^{\prime}\right)\right) \in \operatorname{Bim}(T, L, \mu),\left(\theta_{1}, \theta_{2}\right) \in U(L, T)$,

CM1)

$$
\begin{aligned}
\triangle\left[\left((\Theta, \Xi),\left(\Theta^{\prime}, \Xi^{\prime}\right)\right) \cdot\left(\theta_{1}, \theta_{2}\right)\right] & =\triangle\left(\Theta \theta_{1}, \theta_{2} \Xi^{\prime}\right) \\
& =\left(\Theta \theta_{1} \mu, \theta_{2} \Xi^{\prime} \mu\right),\left(\mu \Theta \theta_{1}, \mu \theta_{2} \Xi^{\prime}\right) \\
& =\left(\Theta \theta_{1} \mu, \theta_{2} \mu \Xi\right),\left(\Theta^{\prime} \mu \theta_{1}, \mu \theta_{2} \Xi^{\prime}\right) \\
& =\left(\left(\Theta \circ \Theta_{\theta}, \Xi_{\theta} \circ \Xi\right),\left(\Theta^{\prime} \circ \Theta_{\theta}^{\prime}, \Xi_{\theta}^{\prime} \circ \Xi^{\prime}\right)\right) \\
& =\left[\left((\Theta, \Xi) \circ\left(\Theta_{\theta}, \Xi_{\theta}\right)\right),\left(\left(\Theta^{\prime}, \Xi^{\prime}\right) \circ\left(\Theta_{\theta}^{\prime}, \Xi_{\theta}^{\prime}\right)\right)\right] \\
& =\left((\Theta, \Xi),\left(\Theta^{\prime}, \Xi^{\prime}\right)\right) \circ\left(\left(\Theta_{\theta}^{\prime}, \Xi_{\theta}^{\prime}\right),\left(\Theta_{\theta}, \Xi_{\theta}\right)\right) \\
& =\left((\Theta, \Xi),\left(\Theta^{\prime}, \Xi^{\prime}\right)\right) \triangle\left(\theta_{1}, \theta_{2}\right)
\end{aligned}
$$




$$
\begin{aligned}
\triangle\left[\left(\theta_{1}, \theta_{2}\right) \cdot\left((\Theta, \Xi),\left(\Theta^{\prime}, \Xi^{\prime}\right)\right)\right] & =\triangle\left(\theta_{1} \Theta^{\prime}, \Xi \theta_{2}\right) \\
& =\left(\theta_{1} \Theta^{\prime} \mu, \Xi \theta_{2} \mu\right),\left(\mu \theta_{1} \Theta^{\prime}, \mu \Xi \theta_{2}\right) \\
& =\left(\theta_{1} \mu \Theta, \Xi \theta_{2} \mu\right),\left(\mu \theta_{1} \Theta^{\prime}, \Xi^{\prime} \mu \theta_{2}\right) \\
& =\left(\left(\Theta_{\theta} \circ \Theta, \Xi \circ \Xi_{\theta}\right),\left(\Theta_{\theta}^{\prime} \circ \Theta^{\prime}, \Xi^{\prime} \circ \Xi_{\theta}^{\prime}\right)\right) \\
& =\left[\left(\left(\Theta_{\theta}, \Xi_{\theta}\right) \circ(\Theta, \Xi)\right),\left(\left(\Theta_{\theta}^{\prime}, \Xi_{\theta}^{\prime}\right) \circ\left(\Theta^{\prime}, \Xi^{\prime}\right)\right)\right] \\
& =\left(\left(\Theta_{\theta}^{\prime}, \Xi_{\theta}^{\prime}\right),\left(\Theta_{\theta}, \Xi_{\theta}\right)\right) \circ\left((\Theta, \Xi),\left(\Theta^{\prime}, \Xi^{\prime}\right)\right) \\
& =\triangle\left(\theta_{1}, \theta_{2}\right) \circ\left((\Theta, \Xi),\left(\Theta^{\prime}, \Xi^{\prime}\right)\right)
\end{aligned}
$$

CM2)

$$
\begin{aligned}
\triangle\left(\beta_{1}, \beta_{2}\right) \cdot\left(\theta_{1}, \theta_{2}\right) & =\left(\left(\Theta_{\beta}, \Xi_{\beta}\right),\left(\Theta_{\beta}^{\prime}, \Xi_{\beta}^{\prime}\right)\right) \cdot\left(\theta_{1}, \theta_{2}\right) \\
& =\left(\Theta_{\beta} \theta_{1}, \theta_{2} \Xi_{\beta}^{\prime}\right) \\
& =\left(\beta_{1} \mu \theta_{1}, \theta_{2} \mu \beta_{2}\right) \\
& =\left(\beta_{1}, \beta_{2}\right) \circ\left(\theta_{1}, \theta_{2}\right) \\
\left(\beta_{1}, \beta_{2}\right) \cdot \triangle\left(\theta_{1}, \theta_{2}\right) & =\left(\beta_{1}, \beta_{2}\right) \cdot\left(\left(\Theta_{\theta}, \Xi_{\theta}\right),\left(\Theta_{\theta}^{\prime}, \Xi_{\theta}^{\prime}\right)\right) \\
& =\left(\beta_{1} \Theta_{\theta}^{\prime}, \Xi_{\theta} \beta_{2}\right) \\
& =\left(\beta_{1} \mu \theta_{1}, \theta_{2} \mu \beta_{2}\right) \\
& =\left(\beta_{1}, \beta_{2}\right) \circ\left(\theta_{1}, \theta_{2}\right)
\end{aligned}
$$

The crossed module $(U(L, T), \operatorname{Bim}(T, L, \mu), \triangle)$ will be called the "the actor crossed module" of $(T, L, \mu)$ and it will be denoted by $\mathcal{A}(T, L, \mu)$.

Lemma 3.10. If $\mu: T \longrightarrow L$ is a crossed module, then $\eta: T \rightarrow U(L, T)$ given by $\eta(t)=\left(\eta_{1 t}, \eta_{2 t}\right)$ is an algebra morphism where $\eta_{1 t}(l)=t \cdot l, \eta_{2 t}(l)=l \cdot t$ and $\eta(T)$ is an ideal of $U(L, T)$.

Lemma 3.11.

$$
\begin{aligned}
\alpha: L & \rightarrow \operatorname{Bim}(T, L, \mu) \\
l & \mapsto\left(\left(\Theta_{l}, \Xi_{l}\right),\left(\Theta_{l}^{\prime}, \Xi_{l}^{\prime}\right)\right)
\end{aligned}
$$

is an algebra morphism where $\Theta_{l}(t)=l \cdot t, \Theta_{l}^{\prime}\left(l^{\prime}\right)=l l^{\prime}, \Xi_{l}(t)=t \cdot l, \Xi_{l}^{\prime}\left(l^{\prime}\right)=l^{\prime} l$, and $\alpha(L)$ is an ideal of $\operatorname{Bim}(T, L, \mu)$.

Theorem 3.12. If $\mu: T \longrightarrow L$ is a crossed module, then the morphism

$$
(\eta, \alpha):(T, L, \mu) \rightarrow \mathcal{A}(T, L, \mu)
$$

is a morphism of crossed modules where $(\eta(t))(l)=\eta_{t}(l)=\left(\eta_{1 t}(l), \eta_{2 t}(l)\right)$ and $\alpha(l)=\left(\left(\Theta_{l}, \Xi_{l}\right),\left(\Theta_{l}^{\prime}, \Xi_{l}^{\prime}\right)\right)$. Proof.

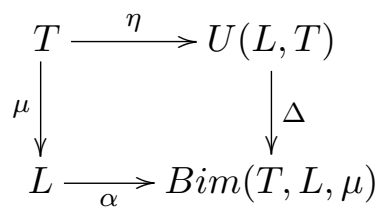


$i$.

$$
\begin{aligned}
\triangle \eta(t) & =\Delta \eta_{t}(l) \\
& =\triangle\left(\eta_{1 t}(l), \eta_{2 t}(l)\right) \\
& =\left[\left(\Theta_{\eta_{1 t}}\left(t^{\prime}\right), \Xi_{\eta_{2 t}}\left(t^{\prime}\right)\right),\left(\Theta_{\eta_{1 t}}^{\prime}(l), \Xi_{\eta_{2 t}}^{\prime}(l)\right)\right] \\
& =\left[\left(\eta_{1 t} \mu\left(t^{\prime}\right), \eta_{2 t} \mu\left(t^{\prime}\right)\right),\left(\mu \eta_{1 t}(l), \mu \eta_{2 t}(l)\right)\right] \\
& =\left[\left(t \cdot \mu\left(t^{\prime}\right), \mu\left(t^{\prime}\right) \cdot t\right),(\mu(t \cdot l), \mu(l \cdot t))\right] \\
& =\left[\left(\mu(t) \cdot t^{\prime}, t^{\prime} \cdot \mu(t)\right),(\mu(t) l, l \mu(t))\right] \\
& =\left[\left(\Theta_{\mu(t)}, \Xi_{\mu(t)}\right),\left(\Theta_{\mu(t)}^{\prime}, \Xi_{\mu(t)}^{\prime}\right)\right] \\
& =\alpha(\mu(t)) \\
& =\alpha \mu(t)
\end{aligned}
$$

ii.

$$
\begin{aligned}
(\alpha(l) \circ \eta(t))\left(l^{\prime}\right) & \left.=\left[\left(\Theta_{l}, \Xi_{l}\right),\left(\Theta_{l}^{\prime}, \Xi_{l}^{\prime}\right)\right) \cdot\left(\eta_{1 t}, \eta_{2 t}\right)\right]\left(l^{\prime}\right) \\
& =\left(\Theta_{l} \eta_{1 t}, \eta_{2 t} \Xi_{l}^{\prime}\right)\left(l^{\prime}\right) \\
& =\left[\Theta_{l} \eta_{1 t}\left(l^{\prime}\right), \eta_{2 t} \Xi_{l}^{\prime}\left(l^{\prime}\right)\right] \\
& =\left(\Theta_{l}\left(t \cdot l^{\prime}\right), \eta_{2 t}\left(l^{\prime} l\right)\right) \\
& =\left(l \cdot\left(t \cdot l^{\prime}\right),\left(l^{\prime} l\right) \cdot t\right) \\
& =\left((l \cdot t) \cdot l^{\prime}, l^{\prime} \cdot(l \cdot t)\right) \\
& =\left(\eta_{1 l \cdot t}\left(l^{\prime}\right), \eta_{2 l \cdot t}\left(l^{\prime}\right)\right) \\
& =\eta(l \cdot t)\left(l^{\prime}\right)
\end{aligned}
$$

and

$$
\begin{aligned}
(\eta(t) \circ \alpha(l))\left(l^{\prime}\right) & =\left[\left(\eta_{1 t}, \eta_{2 t}\right) \cdot\left(\left(\Theta_{l}, \Xi_{l}\right),\left(\Theta_{l}^{\prime}, \Xi_{l}^{\prime}\right)\right)\right]\left(l^{\prime}\right) \\
& =\left(\eta_{1 t} \Theta_{l}^{\prime}, \Xi_{l} \eta_{2 t}\right)\left(l^{\prime}\right) \\
& =\left[\eta_{1 t} \Theta_{l}^{\prime}\left(l^{\prime}\right), \Xi_{l} \eta_{2 t}\left(l^{\prime}\right)\right] \\
& =\left(\eta_{1 t}\left(l^{\prime} l\right), \Xi_{l}\left(l^{\prime} \cdot t\right)\right) \\
& =\left(\eta_{1 t}\left(l^{\prime}\right) \cdot l,\left(l^{\prime} \cdot t\right) \cdot l\right) \\
& =\left((t \cdot l) \cdot l^{\prime},\left(l^{\prime} \cdot t\right) \cdot l\right) \\
& =\left((t \cdot l) \cdot l^{\prime}, l^{\prime} \cdot(t \cdot l)\right) \\
& =\left(\eta_{1 t \cdot l}\left(l^{\prime}\right), \eta_{2 t \cdot l}\left(l^{\prime}\right)\right) \\
& =\eta(t \cdot l)\left(l^{\prime}\right)
\end{aligned}
$$

Proposition 3.13. $\operatorname{Im}(\eta, \alpha)=(\operatorname{Im}(\eta), \operatorname{Im}(\alpha))$ is an ideal of $(\mathcal{U}(L, T), \operatorname{Bim}(T, L, \mu), \Delta)$.

\section{Proof.}

i. For $\left((\Theta, \Xi),\left(\Theta^{\prime}, \Xi^{\prime}\right)\right) \in \operatorname{Bim}(T, L, \mu),\left(\left(\Theta_{l}, \Xi_{l}\right),\left(\Theta_{l}^{\prime}, \Xi_{l}^{\prime}\right)\right) \in \operatorname{Im}(\alpha)$,

$$
\begin{aligned}
\left(\left(\Theta_{l}, \Xi_{l}\right),\left(\Theta_{l}^{\prime}, \Xi_{l}^{\prime}\right)\right) \circ\left((\Theta, \Xi),\left(\Theta^{\prime}, \Xi^{\prime}\right)\right) & =\left(\left(\Theta_{l} \circ \Theta, \Xi \circ \Xi_{l}\right),\left(\Theta_{l}^{\prime} \circ \Theta^{\prime}, \Xi^{\prime} \circ \Xi_{l}^{\prime}\right)\right) \\
& =\left(\left(\Theta_{\Xi^{\prime}(l)}, \Xi_{\Xi^{\prime}(l)}\right),\left(\Theta_{\Xi^{\prime}(l)}^{\prime}, \Xi_{\Xi^{\prime}(l)}^{\prime}\right)\right) \in \operatorname{Im}(\alpha)
\end{aligned}
$$

and

$$
\left((\Theta, \Xi),\left(\Theta^{\prime}, \Xi^{\prime}\right)\right) \circ\left(\left(\Theta_{l}, \Xi_{l}\right),\left(\Theta_{l}^{\prime}, \Xi_{l}^{\prime}\right)\right)=\left(\left(\Theta_{\Theta^{\prime}(l)}, \Xi_{\Theta^{\prime}(l)}\right),\left(\Theta_{\Theta^{\prime}(l)}^{\prime}, \Xi_{\Theta^{\prime}(l)}^{\prime}\right)\right) \in \operatorname{Im}(\alpha)
$$


ii. For $\left((\Theta, \Xi),\left(\Theta^{\prime}, \Xi^{\prime}\right)\right) \in \operatorname{Bim}(T, L, \mu),\left(\eta_{1 t}, \eta_{2 t}\right) \in \operatorname{Im}(\eta)$, since

$$
\left[\left((\Theta, \Xi),\left(\Theta^{\prime}, \Xi^{\prime}\right)\right) \cdot\left(\eta_{1 t}, \eta_{2 t}\right)\right](l)=\left(\eta_{1 \Theta(t)}, \eta_{2 \Theta(t)}\right)(l)
$$

and

$$
\left[\left(\eta_{1 t}, \eta_{2 t}\right) \cdot\left((\Theta, \Xi),\left(\Theta^{\prime}, \Xi^{\prime}\right)\right)\right](l)=\left(\eta_{1 \Xi(t)}, \eta_{2 \Xi(t)}\right)(l)
$$

We get $\left(\eta_{1 \Theta(t)}, \eta_{2 \Theta(t)}\right)$ and $\left(\eta_{1 \Xi(t)}, \eta_{2 \Xi(t)}\right)$ belong to $\operatorname{Im}(\eta)$.

iii. For $\left(\left(\Theta_{l}, \Xi_{l}\right),\left(\Theta_{l}^{\prime}, \Xi_{l}^{\prime}\right)\right) \in \operatorname{Im}(\alpha),\left(\beta_{1}, \beta_{2}\right) \in U(L, T), l^{\prime} \in L$, since

$$
\begin{aligned}
{\left[\left(\left(\Theta_{l}, \Xi_{l}\right),\left(\Theta_{l}^{\prime}, \Xi_{l}^{\prime}\right)\right) \cdot\left(\beta_{1}, \beta_{2}\right)\right]\left(l^{\prime}\right) } & =\left(\Theta_{l} \beta_{1}, \beta_{2} \Xi_{l}^{\prime}\right)\left(l^{\prime}\right) \\
& =\left(\Theta_{l} \beta_{1}\left(l^{\prime}\right), \beta_{2} \Xi_{l}^{\prime}\left(l^{\prime}\right)\right) \\
& =\left(l \cdot \beta_{1}\left(l^{\prime}\right), \beta_{2}\left(l^{\prime} l\right)\right) \\
& =\left(l \cdot \beta_{1}\left(l^{\prime}\right), l^{\prime} \cdot \beta_{2}(l)\right) \\
& =\left(\beta_{2}(l) \cdot l^{\prime}, l^{\prime} \cdot \beta_{2}(l)\right) \\
& =\left(\eta_{1 \beta_{2}(l)}\left(l^{\prime}\right), \eta_{2 \beta_{2}(l)}\left(l^{\prime}\right)\right) \\
{\left[\left(\beta_{1}, \beta_{2}\right) \cdot\left(\left(\Theta_{l}, \Xi_{l}\right),\left(\Theta_{l}^{\prime}, \Xi_{l}^{\prime}\right)\right)\right]\left(l^{\prime}\right) } & =\left(\beta_{1} \Theta_{l}^{\prime}, \Xi_{l} \beta_{2}\right)\left(l^{\prime}\right) \\
& =\left(\beta_{1}\left(\Theta_{l}^{\prime}\left(l^{\prime}\right)\right), \Xi_{l}\left(\beta_{2}\left(l^{\prime}\right)\right)\right) \\
& =\left(\beta_{1}\left(l l^{\prime}\right), \beta_{2}\left(l^{\prime}\right) \cdot l\right) \\
& =\left(\beta_{1}(l) \cdot l^{\prime}, l^{\prime} \cdot \beta_{1}(l)\right) \\
& =\left(\eta_{1 \beta_{1}(l)}\left(l^{\prime}\right), \eta_{2 \beta_{1}(l)}\left(l^{\prime}\right)\right),
\end{aligned}
$$

then

$$
\left[\left(\left(\Theta_{l}, \Xi_{l}\right),\left(\Theta_{l}^{\prime}, \Xi_{l}^{\prime}\right)\right) \cdot\left(\beta_{1}, \beta_{2}\right)\right]=\left(\eta_{1 \beta_{2}(l)}, \eta_{2 \beta_{2}(l)}\right)
$$

and

$$
\left(\beta_{1}, \beta_{2}\right) \cdot\left(\left(\Theta_{l}, \Xi_{l}\right),\left(\Theta_{l}^{\prime}, \Xi_{l}^{\prime}\right)\right)=\left(\eta_{1 \beta_{1}(l)}, \eta_{2 \beta_{1}(l)}\right) \in \operatorname{Im}(\alpha)
$$

\section{The Annihilator of a Crossed Module}

In this section, our object is to generalise the notion of annihilator in associative algebras by using actor crossed module.

Definition 4.1. Let $(T, L, \mu)$ be a crossed module. An annihilator of the crossed module in associative algebras is the kernel of the homomorphism

$$
(\eta, \alpha):(T, L, \mu) \rightarrow \mathcal{A}(T, L, \mu)
$$

and it is denoted by $\operatorname{Ann}(T, L, \mu)$.

$$
A n n(T, L, \mu)=\operatorname{Ker}(\eta, \alpha)=\left(A n n_{T}(L), A n n_{L}(T) \cap A n n_{L}(L), \mu\right)
$$

where

$$
\operatorname{Ker} \eta=\left\{t \in T \mid \eta_{1}(t)=0, \eta_{2}(t)\right\}=\{t \in T \mid t \cdot l=0, l \cdot t=0, l \in L\}=\operatorname{Ann}_{T}(L)
$$

and

$$
\begin{aligned}
\operatorname{Ker} \alpha & =\left\{l \in L \mid \alpha(l)=\left(\left(\Theta_{l}, \Xi_{l}\right),\left(\Theta_{l}^{\prime}, \Xi_{l}^{\prime}\right)\right)=(0,0)\right\} \\
& =\left\{l \in L \mid \Theta_{l}(t)=l \cdot t=0, \Xi_{l}(t)=t \cdot l=0, \Theta_{l}^{\prime}\left(l^{\prime}\right)=l l^{\prime}=0, \Xi_{l}^{\prime}\left(l^{\prime}\right)=l^{\prime} l=0, t \in T, l^{\prime} \in L\right\} \\
& =\operatorname{Ann}_{L}(T) \cap A n n_{L}(L)
\end{aligned}
$$

$\operatorname{Ann}(T, L, \mu)$ is a two sided ideal of the crossed module $\mu: T \longrightarrow L$, because it is the kernel of a crossed module morphism under certain conditions. 


\section{An Application for Crossed Squares}

It is considered that a crossed $n$-cubes is a higher dimensional analogous of crossed modules. If we take the case $n=2$, a crossed square is obtained. As an associative algebra $G$ gives rise to a crossed module $\mu: G \rightarrow \operatorname{Bim}(G)$, that idea allows us to get an generalisation for higher dimension.

The definition of a crossed square in associative algebras is given by Ellis in [12]. Some related studies are [15], [16], and [17].

\subsection{Crossed Squares}

If a commutative diagram of associative algebras

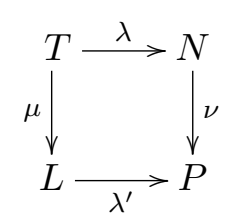

satisfies the following axioms together with functions $h: L \times N \rightarrow T, h^{\prime}: N \times L \rightarrow T$ and actions of $P$ on $T, L$ and $N$, then it is called as a crossed square:

i. The maps $\mu, \lambda, \lambda^{\prime}, \nu$ and $\nu \lambda$ are crossed modules.

ii. The maps $\lambda, \mu$ preserve the action of $P$.

iii. $k h(l, n)=h(k l, n)=h(l, k n)$

$k h^{\prime}(n, l)=h^{\prime}(k n, l)=h^{\prime}(n, k l)$

iv. $p \cdot h(l, n)=h(p \cdot l, n)$

$h(l, n) \cdot p=h(l, n \cdot p)$

$h(l \cdot p, n)=h(l, n \cdot p)$

$p \cdot h^{\prime}(n, l)=h^{\prime}(p \cdot n, l)$

$h^{\prime}(n, l) \cdot p=h^{\prime}(n, l \cdot p)$

$h^{\prime}(n \cdot p, l)=h^{\prime}(n, p \cdot l)$

v. $h\left(l_{1}+l_{2}, n\right)=h\left(l_{1}, n\right)+h\left(l_{2}, n\right)$

$h\left(l, n_{1}+n_{2}\right)=h\left(l, n_{1}\right)+h\left(l, n_{2}\right)$

$h^{\prime}\left(n_{1}+n_{2}, l\right)=h^{\prime}\left(n_{1}, l\right)+h^{\prime}\left(n_{2}, l\right)$

$h^{\prime}\left(n, l_{1}+l_{2}\right)=h^{\prime}\left(n, l_{1}\right)+h^{\prime}\left(n, l_{2}\right)$

vi. $\lambda h(l, n)=\lambda^{\prime} l \cdot n, \lambda h^{\prime}(n, l)=n \cdot \lambda^{\prime} l$

$\lambda^{\prime} h(l, n)=l \cdot \nu n, \lambda^{\prime} h^{\prime}(n, l)=\nu n \cdot l$

vii. $h(l, \lambda t)=\lambda^{\prime} l \cdot t, h^{\prime}(\lambda t, l)=t \cdot \lambda^{\prime} l$

$h(\mu t, n)=t \cdot \nu n, h^{\prime}(n, \mu t)=\nu n \cdot t$

viii. $n_{2} \cdot h\left(l, n_{1}\right)=h^{\prime}\left(n_{2}, l\right) \cdot n_{2}$

$l_{2} \cdot h^{\prime}\left(n, l_{1}\right)=h\left(l_{2}, n\right) \cdot l_{1}$ 
for all $l, l_{1}, l_{2} \in L, n, n_{1}, n_{2} \in N, p \in P, t \in T, k \in K$, where $l \cdot t$ means $\lambda^{\prime}(l) \cdot t$. There are actions of $L$ on $T$ and $N$, via $\lambda^{\prime}$, and of $N$ on $T$ and $L$ via $\nu$.

As a crossed module $\mu: G \rightarrow \operatorname{Bim}(G)$ arises from an associative algebra $G$, we have been able to deduce the result shown below:

Theorem 5.1. If $\mu: T \longrightarrow L$ is a crossed module, then the morphism

$$
(\eta, \alpha):(T, L, \mu) \rightarrow \mathcal{A}(T, L, \mu)
$$

gives rise to the crossed square

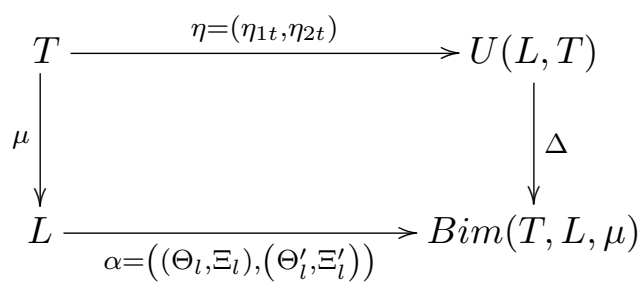

with functions

$$
\begin{aligned}
h: U(L, T) \times L & \rightarrow T \\
\left(\left(\beta_{1}, \beta_{2}\right), l\right) & \mapsto h\left(\left(\beta_{1}, \beta_{2}\right), l\right)=\beta_{1}(l)
\end{aligned}
$$

and

$$
\begin{aligned}
h^{\prime}: L \times U(L, T) & \rightarrow T \\
\left(l,\left(\beta_{1}, \beta_{2}\right)\right) & \mapsto h^{\prime}\left(l,\left(\beta_{1}, \beta_{2}\right)\right)=\beta_{2}(l)
\end{aligned}
$$

where $\operatorname{Bim}(T, L, \mu)$ acts on $L$ and $T$ via the appropriate projections.

\section{ProOF.}

i. $\triangle: U(L, T) \longrightarrow \operatorname{Bim}(T, L, \mu)$ is a crossed module as mentioned before. Since CM1)

$$
\begin{aligned}
\alpha\left(\left((\Theta, \Xi),\left(\Theta^{\prime}, \Xi^{\prime}\right)\right) \cdot l\right) & =\alpha\left(\Theta_{l}^{\prime}\right) \\
& =\left(\left(\Theta_{\Theta_{l}^{\prime}}, \Xi_{\Theta_{l}^{\prime}}\right),\left(\Theta_{\Theta_{l}^{\prime}}^{\prime}, \Xi_{\Theta_{l}^{\prime}}^{\prime}\right)\right) \\
& =\left((\Theta, \Xi),\left(\Theta^{\prime}, \Xi^{\prime}\right)\right) \circ\left(\left(\Theta_{l}, \Xi_{l}\right),\left(\Theta_{l}^{\prime}, \Xi_{l}^{\prime}\right)\right) \\
& =\left((\Theta, \Xi),\left(\Theta^{\prime}, \Xi^{\prime}\right)\right) \circ \alpha(l)
\end{aligned}
$$

and

$$
\begin{aligned}
\alpha\left(l \cdot\left((\Theta, \Xi),\left(\Theta^{\prime}, \Xi^{\prime}\right)\right)\right) & =\alpha\left(\Xi_{l}^{\prime}\right) \\
& =\left(\left(\Theta_{\Xi_{l}^{\prime}}, \Xi_{\Xi_{l}^{\prime}}\right),\left(\Theta_{\Xi_{l}^{\prime}}^{\prime}, \Xi_{\Xi_{l}^{\prime}}^{\prime}\right)\right) \\
& =\left(\left(\Theta_{l}, \Xi_{l}\right),\left(\Theta_{l}^{\prime}, \Xi_{l}^{\prime}\right)\right) \circ\left((\Theta, \Xi),\left(\Theta^{\prime}, \Xi^{\prime}\right)\right) \\
& =\alpha(l) \circ\left((\Theta, \Xi),\left(\Theta^{\prime}, \Xi^{\prime}\right)\right)
\end{aligned}
$$

CM2)

$$
\begin{aligned}
\alpha(l) \cdot l^{\prime} & =\left(\left(\Theta_{l}, \Xi_{l}\right),\left(\Theta l^{\prime}, \Xi_{l}^{\prime}\right)\right) \cdot l^{\prime} \\
& =\Theta_{l}^{\prime}\left(l^{\prime}\right)=l l^{\prime}
\end{aligned}
$$

and

$$
\begin{aligned}
l \cdot \alpha\left(l^{\prime}\right) & =l \cdot\left(\left(\Theta_{l^{\prime}}, \Xi l^{\prime}\right),\left(\Theta_{l^{\prime}}^{\prime}, \Xi_{l^{\prime}}^{\prime}\right)\right) \\
& =\Xi_{l^{\prime}}(l)=l l^{\prime},
\end{aligned}
$$

for $\left((\Theta, \Xi),\left(\Theta^{\prime}, \Xi^{\prime}\right)\right) \in \operatorname{Bim}(T, L, \mu), l \in L, \alpha$ is a crossed module.

Also $\chi=\alpha \mu=\Delta \eta$ is a crossed module, because 
CM1)

$$
\begin{aligned}
\chi\left(\left((\Theta, \Xi),\left(\Theta^{\prime}, \Xi^{\prime}\right)\right) \cdot t\right) & =\chi\left(\Theta_{t}\right) \\
& =\alpha \mu\left(\Theta_{t}\right) \\
& =\left(\left(\Theta_{\mu \Theta_{t}}, \Xi_{\mu \Theta_{t}}\right),\left(\Theta_{\mu \Theta_{t}}^{\prime}, \Xi_{\mu \Theta_{t}}^{\prime}\right)\right) \\
& =\left(\left(\Theta_{\Theta^{\prime} \mu_{t}}, \Xi_{\Theta^{\prime} \mu_{t}}\right),\left(\Theta_{\Theta^{\prime} \mu_{t}}^{\prime}, \Xi_{\Theta^{\prime} \mu_{t}}^{\prime}\right)\right) \\
& =\left((\Theta, \Xi),\left(\Theta^{\prime}, \Xi^{\prime}\right)\right) \circ\left(\left(\Theta_{\mu_{t}}, \Xi_{\mu_{t}}\right),\left(\Theta_{\mu_{t}}^{\prime}, \Xi_{\mu_{t}}^{\prime}\right)\right) \\
& =\left((\Theta, \Xi),\left(\Theta^{\prime}, \Xi^{\prime}\right)\right) \circ \alpha \mu(t) \\
& =\left((\Theta, \Xi),\left(\Theta^{\prime}, \Xi^{\prime}\right)\right) \propto \chi(t)
\end{aligned}
$$

and

$$
\begin{aligned}
\left.\chi\left(t \cdot(\Theta, \Xi),\left(\Theta^{\prime}, \Xi^{\prime}\right)\right)\right) & =\chi\left(\Xi_{t}\right) \\
& =\alpha \mu\left(\Xi_{t}\right) \\
& =\left(\left(\Theta_{\mu \Xi_{t}}, \Xi_{\mu \Xi_{t}}\right),\left(\Theta_{\mu \Xi_{t}}^{\prime}, \Xi_{\mu \Xi_{t}}^{\prime}\right)\right) \\
& =\left(\left(\Theta_{\Xi^{\prime} \mu_{t}}, \Xi_{\Xi^{\prime} \mu_{t}}\right),\left(\Theta_{\Xi^{\prime} \mu_{t}}^{\prime}, \Xi_{\Xi^{\prime} \mu_{t}}^{\prime}\right)\right) \\
& =\left(\left(\Theta_{\mu_{t}}, \Xi_{\mu_{t}}\right),\left(\Theta_{\mu_{t}}^{\prime}, \Xi_{\mu_{t}}^{\prime}\right)\right) \circ\left((\Theta, \Xi),\left(\Theta^{\prime}, \Xi^{\prime}\right)\right) \\
& =\alpha \mu(t) \circ\left((\Theta, \Xi),\left(\Theta^{\prime}, \Xi^{\prime}\right)\right) \\
& =\chi(t) \circ\left((\Theta, \Xi),\left(\Theta^{\prime}, \Xi^{\prime}\right)\right)
\end{aligned}
$$

CM2)

$$
\begin{aligned}
\chi(t) \cdot t^{\prime} & =\alpha \mu(t) \cdot t^{\prime} \\
& =\left(\left(\Theta_{\mu_{t}}, \Xi_{\mu_{t}}\right),\left(\Theta_{\mu_{t}}^{\prime}, \Xi_{\mu_{t}}^{\prime}\right)\right) \cdot t^{\prime} \\
& =\Theta_{\mu_{t}}\left(t^{\prime}\right) \\
& =\mu(t) \cdot t^{\prime} \\
& =t t^{\prime}
\end{aligned}
$$

and

$$
\begin{aligned}
t \cdot \chi\left(t^{\prime}\right) & =t \cdot \alpha \mu\left(t^{\prime}\right) \\
& =t \cdot\left(\left(\Theta_{\mu_{t^{\prime}}}, \Xi_{\mu_{t^{\prime}}}\right),\left(\Theta_{\mu_{t^{\prime}}}^{\prime}, \Xi_{\mu_{t^{\prime}}}^{\prime}\right)\right) \\
& =\Xi_{\mu_{t^{\prime}}}(t) \\
& =t \cdot \mu\left(t^{\prime}\right) \\
& =t t^{\prime}
\end{aligned}
$$

for $\left((\Theta, \Xi),\left(\Theta^{\prime}, \Xi^{\prime}\right)\right) \in \operatorname{Bim}(T, L, \mu), t \in T$.

ii. Since

$$
\begin{aligned}
\alpha\left(\left((\Theta, \Xi),\left(\Theta^{\prime}, \Xi^{\prime}\right)\right) \cdot t\right) & =\mu\left(\Theta_{t}\right) \\
& =\Theta^{\prime} \mu(t) \\
& =\left((\Theta, \Xi),\left(\Theta^{\prime}, \Xi^{\prime}\right)\right) \circ \mu(t) \\
\alpha\left(t \cdot\left((\Theta, \Xi),\left(\Theta^{\prime}, \Xi^{\prime}\right)\right)\right) & =\mu\left(\Xi_{t}\right) \\
& =\Xi^{\prime} \mu(t) \\
& =\mu(t) \circ\left((\Theta, \Xi),\left(\Theta^{\prime}, \Xi^{\prime}\right)\right)
\end{aligned}
$$


and

$$
\begin{aligned}
\eta\left(\left((\Theta, \Xi),\left(\Theta^{\prime}, \Xi^{\prime}\right)\right) \cdot t\right) & =\eta\left(\Theta_{t}\right) \\
& =\left(\eta_{1 \Theta_{t}}, \eta_{2 \Theta_{t}}\right) \\
& =\left((\Theta, \Xi),\left(\Theta^{\prime}, \Xi^{\prime}\right)\right) \cdot\left(\eta_{1 t}, \eta_{2 t}\right) \\
& =\left((\Theta, \Xi),\left(\Theta^{\prime}, \Xi^{\prime}\right)\right) \cdot \eta(t) \\
\eta\left(t \cdot\left((\Theta, \Xi),\left(\Theta^{\prime}, \Xi^{\prime}\right)\right)\right) & =\eta\left(\Xi_{c}\right) \\
& =\left(\eta_{1 \Xi_{t}}, \eta_{2 \Xi_{t}}\right) \\
& =\left(\eta_{1 t}, \eta_{2 t}\right) \cdot\left((\Theta, \Xi),\left(\Theta^{\prime}, \Xi^{\prime}\right)\right) \\
& =\eta(t) \cdot\left((\Theta, \Xi),\left(\Theta^{\prime}, \Xi^{\prime}\right)\right)
\end{aligned}
$$

for $\left((\Theta, \Xi),\left(\Theta^{\prime}, \Xi^{\prime}\right)\right) \in \operatorname{Bim}(T, L, \mu), t \in T, \mu$ and $\eta$ preserve the action of $\operatorname{Bim}(T, L, \mu)$.

iii.

$$
\begin{aligned}
k h\left(\left(\beta_{1}, \beta_{2}\right), l\right) & =k\left(\beta_{1}(l)\right) \\
& =k \beta_{1}(l) \\
& =\beta_{1}(k l) \\
& =h\left(\left(\beta_{1}, \beta_{2}\right)(k l)\right) \\
& =k\left(\beta_{1}(l)\right) \\
& =\left(k\left(\beta_{1}\right)\right) l \\
& =h\left(k\left(\beta_{1}, \beta_{2}\right), l\right) \\
k h^{\prime}\left(l,\left(\beta_{1}, \beta_{2}\right)\right) & =k\left(\beta_{2}(l)\right) \\
& =k \beta_{2}(l) \\
& =\beta_{2}(k l) \\
& =h^{\prime}\left((k g),\left(\beta_{1}, \beta_{2}\right)\right) \\
& =k\left(\beta_{2}(l)\right) \\
& =\left(k\left(\beta_{2}\right)\right) l \\
& =h^{\prime}\left(l, k\left(\beta_{1}, \beta_{2}\right)\right)
\end{aligned}
$$

for $k \in K,\left(\beta_{1}, \beta_{2}\right) \in U(L, T), l \in L$.

iv. For $\left((\Theta, \Xi),\left(\Theta^{\prime}, \Xi^{\prime}\right)\right) \in \operatorname{Bim}(T, L, \mu), l \in L$,

$$
\begin{aligned}
\left((\Theta, \Xi),\left(\Theta^{\prime}, \Xi^{\prime}\right)\right) \cdot\left(h\left(\left(\beta_{1}, \beta_{2}\right), l\right)\right) & =\left((\Theta, \Xi),\left(\Theta^{\prime}, \Xi^{\prime}\right)\right) \cdot \beta_{1}(l) \\
& =\Theta_{\beta_{1}(l)} \\
& =\Theta \beta_{1}(l) \\
& =h\left(\left(\Theta \beta_{1}, \beta_{2} \Xi^{\prime}\right), l\right) \\
& \left.=h\left(\left((\Theta, \Xi),\left(\Theta^{\prime}, \Xi^{\prime}\right)\right) \cdot\left(\beta_{1}, \beta_{2}\right)\right), l\right) \\
\left.h\left(\left(\beta_{1}, \beta_{2}\right), l\right)\right) \cdot\left((\Theta, \Xi),\left(\Theta^{\prime}, \Xi^{\prime}\right)\right) & =\beta_{1}(l) \cdot\left((\Theta, \Xi),\left(\Theta^{\prime}, \Xi^{\prime}\right)\right) \\
& =\Xi_{\beta_{1}(l)} \\
& =\Xi \beta_{1}(l) \\
& =\beta_{1} \Xi_{l}^{\prime} \\
& =h\left(\left(\beta_{1}, \beta_{2}\right), \Xi_{l}^{\prime}\right) \\
& =h\left(\left(\beta_{1}, \beta_{2}\right), l \cdot\left((\Theta, \Xi),\left(\Theta^{\prime}, \Xi^{\prime}\right)\right)\right)
\end{aligned}
$$




$$
\begin{aligned}
& \left.\left.h\left(\left((\Theta, \Xi),\left(\Theta^{\prime}, \Xi^{\prime}\right)\right) \cdot\left(\beta_{1}, \beta_{2}\right), l\right)\right)=h\left(\left(\Theta \beta_{1}, \beta_{2} \Xi^{\prime}\right), l\right)\right) \\
& =\Theta_{\beta_{1}(l)} \\
& =\Theta \beta_{1}(l) \\
& =\beta_{1} \Theta^{\prime}(l) \\
& =h\left(\left(\beta_{1} \Theta^{\prime}, \Xi \beta_{2}\right), l\right) \\
& =h\left(\left(\left(\beta_{1}, \beta_{2}\right) \cdot\left(\left(\Theta_{l}, \Xi_{l}\right),\left(\Theta_{l}^{\prime}, \Xi_{l}^{\prime}\right)\right), l\right)\right. \\
& \left((\Theta, \Xi),\left(\Theta^{\prime}, \Xi^{\prime}\right)\right) \cdot\left(h^{\prime}\left(l,\left(\beta_{1}, \beta_{2}\right)\right)\right)=\left((\Theta, \Xi),\left(\Theta^{\prime}, \Xi^{\prime}\right)\right) \cdot \beta_{2}(l) \\
& =\Theta_{\beta_{2}(l)} \\
& =\Theta \beta_{2}(l) \\
& =\beta_{2} \Theta^{\prime}(l) \\
& =h^{\prime}\left(\Theta_{l}^{\prime},\left(\beta_{1}, \beta_{2}\right)\right) \\
& =h^{\prime}\left(\left((\Theta, \Xi),\left(\Theta^{\prime}, \Xi^{\prime}\right)\right) \cdot l,\left(\beta_{1}, \beta_{2}\right)\right) \\
& \left.h^{\prime}\left(\left(\beta_{1}, \beta_{2}\right), l\right)\right) \cdot\left((\Theta, \Xi),\left(\Theta^{\prime}, \Xi^{\prime}\right)\right)=\beta_{2}(l) \cdot\left((\Theta, \Xi),\left(\Theta^{\prime}, \Xi^{\prime}\right)\right) \\
& =\Xi_{\beta_{2}(l)} \\
& =\Xi \beta_{2}(l) \\
& =\beta_{2} \Xi_{l}^{\prime} \\
& =h^{\prime}\left(l,\left(\Theta \beta_{1}, \beta_{2} \Xi^{\prime}\right)\right) \\
& =h^{\prime}\left(l,\left(\beta_{1}, \beta_{2}\right) \cdot\left((\Theta, \Xi),\left(\Theta^{\prime}, \Xi^{\prime}\right)\right)\right) \\
& \left.h^{\prime}\left(\left(\beta_{1}, \beta_{2}\right) \cdot\left(\left(\Theta_{\beta}, \Xi_{\beta}\right),\left(\Theta_{\beta}^{\prime}, \Xi_{\beta}^{\prime}\right)\right), \beta\right)\right)=h^{\prime}\left(\beta ;\left(\beta_{1} \Theta^{\prime}, \Xi \beta_{2}\right)\right) \\
& =\Xi_{\beta_{2}(l)} \\
& =\Xi \beta_{2}(l) \\
& =\beta_{2} \Xi^{\prime}(l) \\
& =h^{\prime}\left(l,\left(\Theta \beta_{1}, \beta_{2} \Xi^{\prime}\right)\right) \\
& =h^{\prime}\left(l,\left(\beta_{1}, \beta_{2}\right) \cdot\left(\left(\Theta_{l}, \Xi_{l}\right),\left(\Theta_{l}^{\prime}, \Xi_{l}^{\prime}\right)\right)\right)
\end{aligned}
$$

$v$.

$$
\begin{aligned}
h\left(\left(\beta_{1}, \beta_{2}\right)+\left(\theta_{1}, \theta_{2}\right), l\right) & =h\left(\left(\beta_{1}+\theta_{1}, \beta_{2}+\theta_{2}\right), l\right) \\
& =\left(\beta_{1}+\theta_{1}\right) l \\
& =\beta_{1}(l)+\theta_{1}(l) \\
& =h\left(\left(\beta_{1}, \beta_{2}\right), l\right)+h\left(\left(\theta_{1}, \theta_{2}\right), l\right) \\
h\left(\left(\beta_{1}, \beta_{2}\right), l_{1}+l_{2}\right)= & \beta_{1}\left(l_{1}+l_{2}\right) \\
= & \beta_{1}\left(l_{1}\right)+\beta_{1}\left(l_{2}\right) \\
= & \left.h\left(\left(\beta_{1}, \beta_{2}\right), l_{1}\right)+h\left(\left(\beta_{1}, \beta_{2}\right)\right), l_{2}\right) \\
h^{\prime}\left(l_{1}+l_{2},\left(\beta_{1}, \beta_{2}\right)\right)= & \beta_{2}\left(l_{1}+l_{2}\right) \\
= & \beta_{2}\left(l_{1}\right)+\beta_{2}\left(l_{2}\right) \\
= & h^{\prime}\left(l_{1},\left(\beta_{1}, \beta_{2}\right)\right)+h^{\prime}\left(l_{2},\left(\beta_{1}, \beta_{2}\right)\right) \\
h^{\prime}\left(l,\left(\beta_{1}, \beta_{2}\right)+\left(\theta_{1}, \theta_{2}\right)\right) & =h^{\prime}\left(l,\left(\beta_{1}+\theta_{1}, \beta_{2}+\theta_{2}\right)\right) \\
& =\left(\beta_{2}+\theta_{2}\right)(l) \\
& =\beta_{2}(g)+\theta_{2}(l) \\
& =h^{\prime}\left(l,\left(\beta_{1}, \beta_{2}\right)\right)+h^{\prime}\left(l,\left(\theta_{1}, \theta_{2}\right)\right)
\end{aligned}
$$


$v i$.

$$
\begin{aligned}
& \mu h\left(\left(\beta_{1}, \beta_{2}\right), l\right)=\mu\left(\beta_{1}(l)\right) \\
& =\mu \beta_{1}(l) \\
& =\left(\left(\beta_{1} \mu, \beta_{2} \mu\right),\left(\mu \beta_{1}, \mu \beta_{2}\right)\right) \cdot l \\
& =\left(\left(\Theta_{\beta}, \Xi_{\beta}\right),\left(\Theta_{\beta}^{\prime}, \Xi_{\beta}^{\prime}\right)\right) \cdot l \\
& =\left(\triangle\left(\beta_{1}, \beta_{2}\right)\right) \cdot l \\
& \mu h^{\prime}\left(l,\left(\beta_{1}, \beta_{2}\right)\right)=\mu\left(\beta_{2}(l)\right) \\
& =\mu \beta_{2}(l) \\
& =l \cdot\left(\left(\beta_{1} \mu, \beta_{2} \mu\right),\left(\mu \beta_{1}, \mu \beta_{2}\right)\right) \\
& =l \cdot\left(\left(\Theta_{\beta}, \Xi_{\beta}\right),\left(\Theta_{\beta}^{\prime}, \Xi_{\beta}^{\prime}\right)\right) \\
& =l \cdot\left(\triangle\left(\beta_{1}, \beta_{2}\right)\right) \\
& \eta h\left(\left(\beta_{1}, \beta_{2}\right), l\right)=\eta\left(\beta_{1}(l)\right) \\
& =\left(\eta_{1 \beta_{1}(l)}, \eta_{2 \beta_{1}(l)}\right) \\
& \stackrel{(*)}{=} \quad\left(\left(\beta_{1} \Theta_{l}^{\prime}, \Xi_{l} \beta_{2}\right)\right. \\
& =\left(\beta_{1}, \beta_{2}\right) \cdot\left(\left(\Theta_{l}, \Xi_{l}\right),\left(\Theta_{l}^{\prime}, \Xi_{l}^{\prime}\right)\right) \\
& =\left(\beta_{1}, \beta_{2}\right) \cdot \alpha(l)
\end{aligned}
$$

$(*)$

$$
\begin{aligned}
& \eta_{1 \beta_{1}(l)}\left(l^{\prime}\right)=\beta_{1}(l) \cdot l^{\prime} \quad \eta_{2 \beta_{1}(l)}\left(l^{\prime}\right)=l^{\prime} \cdot \beta_{1}(l) \\
& =\beta_{1}\left(l l^{\prime}\right) \quad \text { and } \quad=\beta_{2}\left(l l^{\prime}\right) \\
& =\beta_{1} \Theta_{l}^{\prime}\left(l^{\prime}\right) \quad=\Xi_{l} \beta_{2}\left(l^{\prime}\right) \\
& \eta h^{\prime}\left(\left(\beta_{1}, \beta_{2}\right), l\right)=\eta\left(\beta_{2}(l)\right) \\
& =\left(\eta_{1 \beta_{2}(l)}, \eta_{2 \beta_{2}(l)}\right) \\
& \stackrel{(*)}{=}\left(\left(\Theta_{l} \beta_{1}, \beta_{2} \Xi_{l}^{\prime}\right)\right. \\
& =\left(\left(\Theta_{l}, \Xi_{l}\right),\left(\Theta_{l}^{\prime}, \Xi_{l}^{\prime}\right)\right) \cdot\left(\beta_{1}, \beta_{2}\right) \\
& =\alpha(l) \cdot\left(\beta_{1}, \beta_{2}\right)
\end{aligned}
$$

$(*)$

$$
\begin{aligned}
& \eta_{1 \beta_{2}(l)}\left(l^{\prime}\right)=\beta_{2}(l) \cdot l^{\prime} \quad \eta_{2 \beta_{2}(l)}\left(l^{\prime}\right)=l^{\prime} \cdot \beta_{2}(l) \\
& =l \cdot \beta_{1}\left(l^{\prime}\right) \quad \text { and } \quad=\beta_{2}\left(l l^{\prime}\right) \\
& =\Theta_{l} \beta_{1}\left(l^{\prime}\right) \quad=\beta_{2} \Xi_{l}^{\prime}\left(l^{\prime}\right)
\end{aligned}
$$

vii.

$$
\begin{aligned}
h\left(\left(\beta_{1}, \beta_{2}\right), \mu(t)\right) & =\beta_{1}(\mu(t)) \\
& =\beta_{1} \mu(t) \\
& =\left(\left(\beta_{1} \mu, \beta_{2} \mu\right),\left(\mu \beta_{1}, \mu \beta_{2}\right)\right) \cdot t \\
& =\left(\left(\Theta_{\beta}, \Xi_{\beta}\right),\left(\Theta_{\beta}^{\prime}, \Xi_{\beta}^{\prime}\right)\right) \cdot t \\
& =\left(\triangle\left(\beta_{1}, \beta_{2}\right)\right) \cdot t
\end{aligned}
$$




$$
\begin{aligned}
h^{\prime}\left(\mu(t),\left(\beta_{1}, \beta_{2}\right)\right) & =\beta_{2}(\mu(t)) \\
& =\beta_{2} \mu(t) \\
& =t \cdot\left(\left(\beta_{1} \mu, \beta_{2} \mu\right),\left(\mu \beta_{1}, \mu \beta_{2}\right)\right) \\
& =t \cdot\left(\left(\Theta_{\beta}, \Xi_{\beta}\right),\left(\Theta_{\beta}^{\prime}, \Xi_{\beta}^{\prime}\right)\right) \\
& =t \cdot\left(\triangle\left(\beta_{1}, \beta_{2}\right)\right) \\
h(\eta(t), l) & =h\left(\left(\eta_{1 t}, \eta_{2 t}\right), l\right) \\
& =\eta_{1 t}(l) \\
& =t \cdot l \\
& =\Xi_{l}(t) \\
& =t \cdot\left(\left(\Theta_{l}, \Xi_{l}\right),\left(\Theta_{l}^{\prime}, \Xi_{l}^{\prime}\right)\right) \\
& =t \cdot \alpha(l) \\
& =h^{\prime}\left(l,\left(\eta_{1 t}, \eta_{2 t}\right)\right) \\
& =\eta_{2 t}(l) \\
& =l \cdot t \\
& =\Theta_{l}(t) \\
& =\left(\left(\Theta_{l}, \Xi_{l}\right),\left(\Theta_{l}^{\prime}, \Xi_{l}^{\prime}\right)\right) \cdot t \\
& =\alpha(l) \cdot t
\end{aligned}
$$

viii.

$$
\begin{aligned}
l^{\prime} \cdot h\left(\left(\beta_{1}, \beta_{2}\right), l\right) & =l^{\prime} \cdot \beta_{1}(l) \\
& =\beta_{2}\left(l^{\prime}\right) \cdot l \\
& =h^{\prime}\left(l^{\prime},\left(\beta_{1}, \beta_{2}\right)\right) \cdot l \\
\left(\theta_{1}, \theta_{2}\right) \cdot h^{\prime}\left(l,\left(\beta_{1}, \beta_{2}\right)\right) & =\triangle\left(\theta_{1}, \theta_{2}\right) \cdot \beta_{2}(l) \\
& =\theta_{1} \mu \beta_{2}(l) \\
& \stackrel{(*)}{=} \beta_{2} \mu \theta_{1}(l) \\
& =\theta_{1}(l) \cdot \triangle\left(\left(\beta_{1}, \beta_{2}\right)\right. \\
& =h\left(\left(\theta_{1}, \theta_{2}\right), l\right) \cdot\left(\beta_{1}, \beta_{2}\right)
\end{aligned}
$$

$(*)$ Because of $L^{2}=L$, for every $l \in L$, we can take $l=l_{1} l_{2}$.

$$
\begin{aligned}
\theta_{1} \mu \beta_{2}\left(l_{1} l_{2}\right) & =\theta_{1} \mu\left(l_{1} \cdot \beta_{2}\left(l_{2}\right)\right) \\
& =\Theta_{\theta}\left(l_{1} \cdot \beta_{2}\left(l_{2}\right)\right) \\
& =\Theta_{\theta}^{\prime}\left(l_{1}\right) \cdot \beta_{2}\left(l_{2}\right) \\
& =\mu \theta_{1}\left(l_{1}\right) \cdot \beta_{2}\left(l_{2}\right) \\
& =\beta_{2}\left(\left(\mu \theta_{1}\left(l_{1}\right)\right) l_{2}\right) \\
& =\beta_{2}\left(\mu\left(\theta_{1}\left(l_{1}\right) l_{2}\right)\right) \\
& =\beta_{2}\left(\mu\left(\theta_{1}\left(l_{1}\right) \cdot l_{2}\right)\right) \\
& =\beta_{2}\left(\mu\left(\theta_{1}\left(l_{1} l_{2}\right)\right)\right) \\
& =\beta_{2} \mu \theta_{1}\left(l_{1} l_{2}\right)
\end{aligned}
$$




\section{Conclusion}

It is well known that the action of any group on itself is given by a group homomorphism from any group to its automorphism group. In the case of associative algebra, the role of automorphism groups replaces with bimultiplication algebra. It is considered that the concept of a crossed module in associative algebra is a generalisation of the concept of an associative algebra. Thus we can generalise the notion of bimultiplication algebra for a crossed module in associative algebras. Thus, we conclude that in this context the notion of action is given by the actor crossed module which is obtained via bimultiplication crossed module. Also since the annihilator of an associative algebra $\mathrm{A}$ is given by the kernel of algebra morphism $A \rightarrow \operatorname{Bim}(A)$, we get the annihilator crossed module as the kernel of the crossed module morphism $(\eta, \alpha):(T, L, \mu) \rightarrow \mathcal{A}(T, L, \mu)$. Furthermore, we see that this morphism gives rise to a crossed square which is a two-dimensional analogous to crossed module.

\section{Conflicts of Interest}

The authors declare no conflict of interest.

\section{References}

[1] S. M. Lane, Extensions and Obstructions for Rings, Illinois Journal of Mathematics 121 (1958) 316-345.

[2] J. H. C. Whitehead, Combinatorial Homotopy I, Bulletin of the American Mathematical Society 55 (1949) 231-245.

[3] J. H. C. Whitehead, Combinatorial Homotopy II, Bulletin of the American Mathematical Society 55 (1949) 453-456.

[4] S. Lichtenbaum, M. Schlessinger, The Cotangent Complex of a Morphism, Transactions of the American Mathematical Society 128(1) (1967) 41-70.

[5] M. Gerstenhaber, On the Deformation of Rings and Algebras: II, Annals of Mathematics 84(1) (1966) 1-19.

[6] P. Dedecker, A. S. T. Lue, A Nonabelian Two-Dimensional Cohomology for Associative Algebras, Bulletin of the American Mathematical Society 72(6) (1966) 1044-1050.

[7] K. J. Norrie, Actions and Automorphisms of Crossed Modules, Bulletin de la Société Mathématique de France 118 (1990) 129-146.

[8] A. S. T. Lue, Semi-Complete Crossed Modules and Holomorp hs of Groups, Bulletin of the London Mathematical Society 11 (1979) 8-16.

[9] Z. Arvasi, U. Ege, Annihilators, Multipliers and Crossed Modules, Applied Categorical Structures 11 (2003) 487-506.

[10] S. Hürmetli, Bimultiplication of Crossed Modules, PhD Dissertation, Eskişehir Osmangazi University, (2016) Eskişehir, Turkey.

[11] Y. Boyacı, J. M. Casas, T. Datuashvili, E. Ö. Uslu, Actions in Modified Categories of Interest with Application Crossed Modules, Theory and Applications of Categories 30(25) (2015) 882-908.

[12] G. J. Ellis, Higher Dimensional Crossed Modules of Algebras, Journal of Pure and Applied Algebra $52(3)(1988) 277-282$.

[13] A. S. -T. Lue, Non-Abelian cohomology of Associative Algebras, The Quarterly Journal of Mathematics 19(1) (1968) 159-180. 
[14] R. Lavendhomme, Th. Lucas, On Modules and Crossed Modules, Journal of Algebra 179 (1996) 936-963.

[15] Z. Arvasi. Crossed Squares and 2-Crossed Modules of Commutative Algebras, Theory and Applications of Categories 3(7) (1997) 160-181.

[16] Z. Arvasi, E. Ulualan, Homotopical Aspects of Commutative Algebras I: Freeness Conditions for Crossed Squares, Journal of Homotopy and Related Structure 10 (2013) 495-518.

[17] E. S. Yılmaz, K. Yılmaz, On Crossed Squares of Commutative Algebras, Mathematical Sciences and Applications E-Notes (8) 2 (2020) 32-41. 